E1-2004-36

S. A. Karamian ${ }^{1 *}$, J. J. Carroll ${ }^{2}$, J. Adam ${ }^{1,3}$, N. A. Demekhina ${ }^{4}$

\title{
PRODUCTION OF THE ${ }^{178 m 2}$ Hf ISOMER \\ USING A 4.5-GEV ELECTRON ACCELERATOR
}

Submitted to «Nuclear Instruments and Methods A»

${ }^{1}$ Joint Institute for Nuclear Research, Dubna, Moscow Region, 141980, Russia

${ }^{2}$ Youngstown State University, Youngstown, Ohio 44555, USA

${ }^{3}$ Nuclear Physics Institute, Rez, Prague, CZ-25068, Czech Republic

${ }^{4}$ Institute of Physics, Yerevan, 375036, Armenia

*E-mail address: karamian@nrmail.jinr.ru 
с использованием ускорителя электронов на энергию 4,5 ГэВ

При получении долгоживущих изомеров требуются высокопродуктивные методы для накопления количеств, достаточных для создания экспериментальных мишеней. Присутствие ${ }^{178 \mathrm{~m} 2} \mathrm{Hf}$ было обнаружено и измерено с хорошей статистической точностью в образце тантала по гамма-активности после облучения тормозным излучением с граничной энергией 4,5 ГэВ на ереванском синхротроне. Значения интегрального и среднего сечений определены из эксперимента, и затем оценено изомерное отношение, которое сравнивается с ранее известным для реакции $p+$ Та при энергии протонов 660 МэВ. Для обеспечения наиболее чистых экспериментальных условий в настоящем эксперименте использованы относительно тонкие мишень и конвертер. Однако для высокопродуктивных облучений на пучке электронов высокой энергии наиболее целесообразно использовать толстый образец, объединяющий функции конвертера и мишени. Задача оптимизации эксперимента по получению изомера решена аналитически, и наибольший выход вычислен с применением калибровки по отношению к экспериментальному выходу. Определено, что максимальный выход должен составить около $3 \cdot 10^{9}$ ядер ${ }^{178 m 2} \mathrm{Hf}$ в секунду при токе пучка электронов 100 мкА. Это меньше, чем могло быть достигнуто на пучке протонов, однако на практике следует сравнить также полную стоимость и условия радиационной безопасности экспериментов. Настоящие результаты дают основу для численных оценок и сравнений.

Работа выполнена в Лаборатории ядерных реакций им. Г. Н. Флерова ОИЯИ.

Препринт Объединенного института ядерных исследований. Дубна, 2004

Karamian S. A. et al.

Production of the ${ }^{178 m 2} \mathrm{Hf}$ Isomer

E1-2004-36

Using a 4.5-GeV Electron Accelerator

High-productivity methods are required for the accumulation of long-lived isomers in amounts that are sufficient for the creation of experimental targets. A tantalum sample was activated with the Yerevan synchrotron using 4.5-GeV bremsstrahlung and the presence of ${ }^{178 m 2} \mathrm{Hf}$ was detected with good statistical accuracy by $\gamma$-activity measurements. The integrated and mean cross-section values were deduced from the experiment. The isomer-to-ground-state ratio was then estimated and compared with that known for the $p+$ Ta reaction studied at $660 \mathrm{MeV}$. In the present experiment, both the converter and the target were relatively thin for better definition of the experimental conditions. However, an assembly designed for high-productivity irradiations should be thick and the converter can also serve as a target sample when irradiated with a high-energy electron beam. The optimization of the isomer production was solved analytically and the largest estimated yield was determined as calibrated to the experimental yield. The maximum yield of ${ }^{178 \mathrm{~m} 2} \mathrm{Hf}$ was found to be of about $3 \cdot 10^{9} \mathrm{nu}-$ clei/s using an electron beam current of $100 \mu \mathrm{A}$. This is lower than the yield achieved with proton beams, although for a practical comparison the total cost and radiation safety conditions should be considered. The present results provide a basis for numerical estimations.

The investigation has been performed at the Flerov Laboratory of Nuclear Reactions, JINR. 


\section{INTRODUCTION}

Studies of high-spin nuclear isomers can provide important information regarding both a nuclear structure [1,2] and innovative applications [3]. A triggered release of the energy stored in nuclear isomers may permit the creation of pulsed sources of gamma radiation, perhaps even a gamma-ray laser. The specific energy stored by some isomers for long periods of time is much greater than that of standard sources of energy. For instance, the high-spin $K^{\pi}=16^{+}$isomeric state ${ }^{178 m 2} \mathrm{Hf}$ stores a specific energy of about $1.3 \mathrm{MJ} / \mathrm{mg}$ with a half-life of 31 years. To study mechanisms by which a release of this energy might be triggered, a critical requirement is that samples of isomeric atoms must be available in milligram or even larger amounts. It is established that the largest quantity of

${ }^{178 m 2} \mathrm{Hf}$ was produced at Los Alamos with $800 \mathrm{MeV}$ protons from a high-current accelerator (formerly LAMPF). The advantage of this method was the ability to accumulate the isomer as a by-product within a massive tantalum beam dump during the operation of the accelerator for other experiments [4]. However, the isomeric material produced in this way contains a very high activity of other radionuclides and the corresponding gamma background remains high even after chemical isolation of the hafnium fraction. The 1.87 -year-lived isotope ${ }^{172} \mathrm{Hf}$ is the most problematic contaminant. The productivity of ${ }^{178 m 2} \mathrm{Hf}$ in spallation reactions with protons at intermediate energies was systematically studied for Ta, $\mathrm{W}$ and Re targets at Dubna using the $660 \mathrm{MeV}$ synchrocyclotron [5-7]. From those works, some recommendations were deduced as to methods by which to optimize spallation production of the isomer. However, no scheme was proposed by which to overcome the basic disadvantage presented by the accompanying production of high contaminant activities.

Better quality sources of ${ }^{178 m 2} \mathrm{Hf}$ were successfully produced by the ${ }^{176} \mathrm{Yb}\left({ }^{4} \mathrm{He}, 2 n\right)$ reaction using a $36 \mathrm{MeV}{ }^{4} \mathrm{He}$-ion beam and subsequent chemical processing of the irradiated $\mathrm{Yb}$ target [8]. Mass separation was finally performed on the remaining material. The problem of isomer separation from the stable ${ }^{178} \mathrm{Hf}$ ground state has not yet been solved. The production of the long-lived isomer by the $\left({ }^{4} \mathrm{He}, 2 n\right)$ reaction is disadvantaged by a much lower absolute yield as compared to the spallation reaction. It is known [9] that the production reaction ${ }^{179} \mathrm{Hf}(n, 2 n){ }^{178 m 2} \mathrm{Hf}$ has a reasonably good cross-section for $14-\mathrm{MeV}$ incident neutrons. The absolute yield is, however, restricted by practical limitations of neutron generators exploiting the $d+\mathrm{T}$ reaction. 
Recently, an attempt was made to observe the production of ${ }^{178 m 2} \mathrm{Hf}$ in reactor irradiations of a ${ }^{\text {nat }} \mathrm{Hf}$ target. It was assumed that some reasonable yield could be obtained due to the ${ }^{178} \operatorname{Hf}\left(n, n^{\prime}\right)$ reaction with the neutrons in the fission spectrum. So far, only an upper limit for the cross-section has been deduced because of the high remaining activity from other products - more sensitive measurements are expected after a longer cooling time. Nevertheless, the production of ${ }^{179 m 2} \mathrm{Hf}$ by $\left(n, n^{\prime}\right)$ reactions was observed [10].

Here the possibility of producing the ${ }^{178 m 2} \mathrm{Hf}$ isomer was investigated using 4.5-GeV bremsstrahlung from an electron synchrotron. The productivity with photons cannot be expected to be higher than in the previous reactions due to the generally lower cross-section characteristic of photonuclear interactions as compared to hadronic interactions. Still experimental results on photon production of this important isomer have not been reported and the present measurements should be valuable in assessing the feasibility of different production approaches. Above $1.5 \mathrm{GeV}$, the photon interaction is characterized by a cross-section of about $0.12 \mathrm{mb} /$ nucleon. Below this energy, meson-generating resonances are known in the 200 to $800 \mathrm{MeV}$ range and giant multipole resonances for photoabsorption appear for $E_{\gamma} \leqslant 100 \mathrm{MeV}$. The quasi-deuteron mechanism of photoabsorption also makes a significant contribution at $E_{\gamma} \leqslant 400 \mathrm{MeV}$. Overall, the crosssection of photon interactions with the nucleus systematically decreases with increasing $E_{\gamma}$. The bremsstrahlung spectrum produced by high-energy electrons fall as $E_{\gamma}^{-1}$, so the major contribution to the nuclear reaction yield is created by photons with $E_{\gamma} \leqslant 1000 \mathrm{MeV}$. The energy transferred to a heavy nucleus by photon reactions in this range should be comparable to that released in the proton interactions at 800 [4] and $660 \mathrm{MeV}$ [5-7]. As a result, the production of a variety of radionuclides can be expected as occurs with proton-induced spallation. Among the radioactive products, high-spin isomers must be represented and the experimental observation of the long-lived ${ }^{178 m 2} \mathrm{Hf}$ isomer was indeed possible. The present work provides an important test of the isomer-to-ground-state ratio for high-spin isomers formed in high-energy electromagnetic interactions with complex nuclei and an estimation of the efficiency for accumulation of significant amounts of ${ }^{178 m 2} \mathrm{Hf}$ using an electron accelerator.

\section{MEASUREMENTS}

Figure 1 shows a schematic view of the experimental arrangement at the Yerevan 4.5-GeV electron synchrotron. The electron beam was extracted from the storage ring and struck a tungsten converter having a $0.3-\mathrm{mm}$ thickness. Transmitted beam electrons and electron-positron pairs generated at the converter were deflected by a magnetic field while the bremsstrahlung photons continued forward within a very narrow cone. Collimators installed far from the converter 


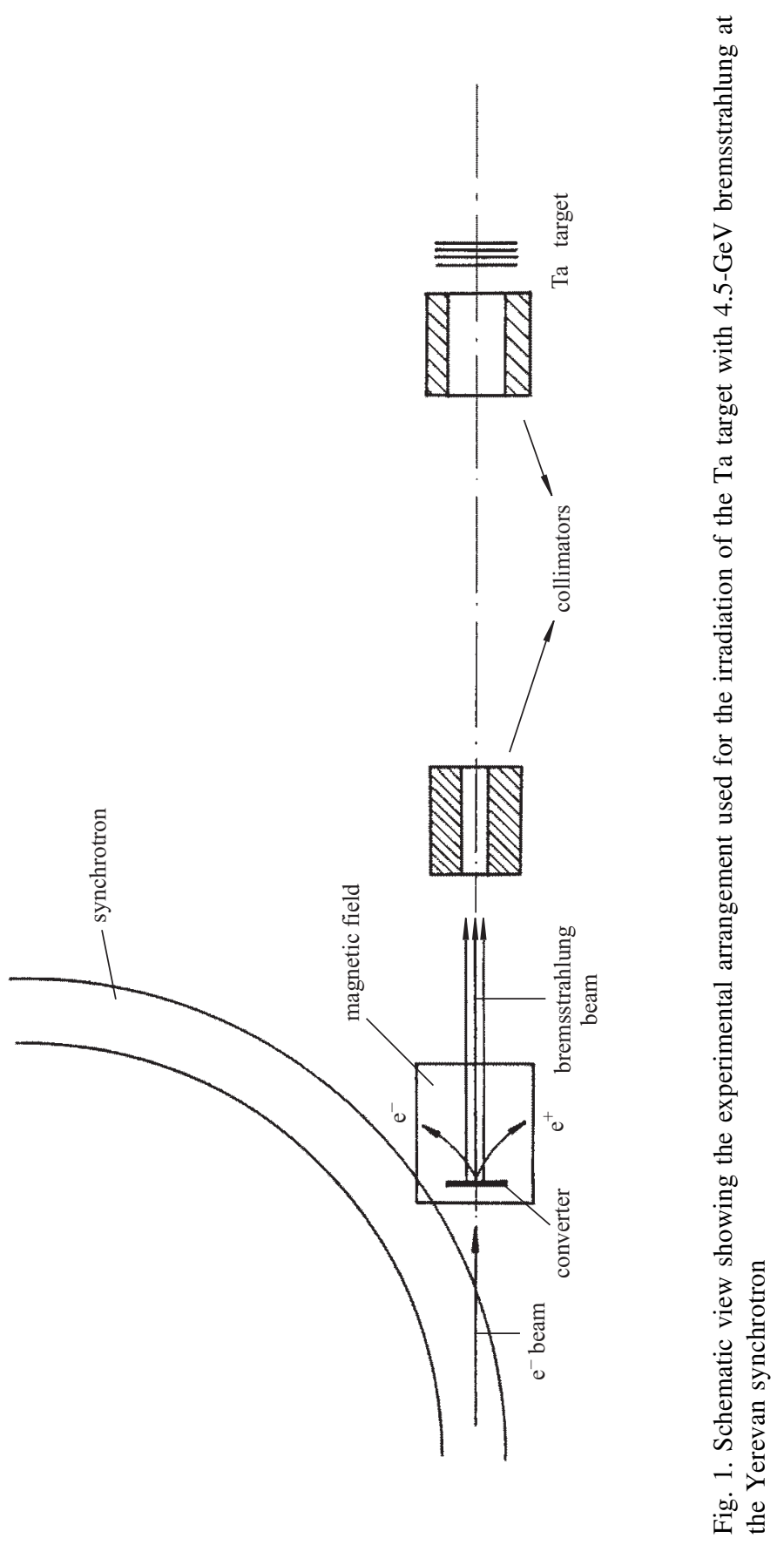


shielded a target from background scattered radiation. The target was made of twenty $0.1-\mathrm{mm}$ thick layers of natural tantalum. The initial electron energy of $4.5 \mathrm{GeV}$ was reduced by about $8 \%$ in the converter and the bremsstrahlung flux was attenuated to a mean value by a factor of about 0.85 due to the absorption within the 2-mm Ta target. These factors were taken into account in the data processing, but were not very pronounced so the approximation of thin converter and thin target could be made to simplify the analysis. The tantalum target was irradiated in 1986 for other purposes and fortunately it was saved up to now, providing the best possible condition for the detection of the 31-year-lived ${ }^{178 m 2} \mathrm{Hf}$ isomer. Many other radionuclides were produced during the irradiation with higher yields than ${ }^{178 m 2} \mathrm{Hf}$, but they have almost decayed completely and did not interfere with the detection of a relatively low activity of ${ }^{178 m 2} \mathrm{Hf}$.

The tantalum target activity was measured using a HPGe spectrometer of $20 \%$ efficiency (relatively to the standard NaI scintillator), combined with compatible electronics to provide an energy resolution of about $1.8 \mathrm{keV}$ for the ${ }^{60} \mathrm{Co} \gamma$ lines. The tantalum foils were distributed to form a two-foil folded layer of $0.2-\mathrm{mm}$ thickness in order to reduce self-absorption of the emitted gamma rays. Even with this arrangement, self-absorption was still not negligible, especially for $E_{\gamma} \leqslant 250 \mathrm{keV}$, and a correction factor was used to take into account the emission efficiency from a sample of finite thickness, $t$ :

$$
\varepsilon=(\mu t)^{-1}\left(1-\mathrm{e}^{-\mu t}\right),
$$

where $\mu\left(E_{\gamma}\right)$ is the linear attenuation coefficient for gammas in tantalum.

Another correction factor to the detector efficiency should account for the finite size (area) of the active source. An efficiency calibration of the Ge detector was done using the normal procedure with standard radioisotope sources of known activity. Such sources are typically small and so can be approximated as point sources. In contrast, the tantalum sample covered an area of about $45 \times 45 \mathrm{~mm}^{2}$ and was installed close to the detector since the low activity from the 31-year isomer required the maximum detection efficiency. The approximation of a point source was not applicable in this case and the solid angle was calculated in order to correct the efficiency. The following equation was used to approximate the mean detection solid angle when activity is uniformly distributed within a circle of radius $R_{s}$ :

$$
\bar{\Omega}=2 \pi\left(\frac{L}{R_{s}}\right)^{2}\left(1-\frac{L}{\sqrt{L^{2}+R_{d}^{2}}}\right) \ln \left[1+\left(\frac{R_{s}}{L}\right)^{2}\right] \text { (sr). }
$$

Here $L$ is the distance between the centers of the source and detector. It was assumed that the sensitive volume of the detector was restricted to the circular entrance window of radius $R_{d}$ and the planes of the source and detector circles were parallel. 
A third correction was needed since the efficiency could be influenced by simultaneous detection of two or more $\gamma$ quanta emitted promptly from a single decay event. In the natural decay cascade of ${ }^{178 m 2} \mathrm{Hf}, 4$ to 5 quanta are emitted and using a close geometry they can generate a sum-signal response in the detector that reduces the intensity of photoabsorption peaks in the $\gamma$-ray spectrum. This is not a random pile-up effect that is dependent on the count rate. Sum signals arise even with weak activity sources whenever the cascade multiplicity $M_{\gamma}>1$ and the solid angle of detection provides sufficient efficiency. Additional measurements were arranged to test the magnitude of this effect for this experiment. The detector efficiency was calibrated at close and far distances using specific point sources that were selected to emit single $\gamma$ quanta. Then, a sample (from other work) that contained a modest activity of ${ }^{178 m 2} \mathrm{Hf}$ was measured at the same locations. The effect of sum signals was significant only when the ${ }^{178 m 2} \mathrm{Hf}$ source was present in close geometry while this effect did not appear in $\gamma$ spectra taken at larger distances or with single-gamma sources. Finally, the deviation of the detector efficiency from the standard values was determined individually for the most important $\gamma$ lines in the decay of ${ }^{178 m 2} \mathrm{Hf}$. These results were used to measure the number of ${ }^{178 m 2} \mathrm{Hf}$ nuclei within the activated tantalum target based on the detected $\gamma$-ray intensity.

With the above-described conditions, a $\gamma$-ray spectrum of the Ta sample which was exposed to $4.5-\mathrm{GeV}$ bremsstrahlung was accumulated during two weeks in order to collect sufficient statistics in the important lines. Fragments of the spectrum are given in Fig. 2 and well-manifested lines of ${ }^{178 m 2} \mathrm{Hf}$ are seen therein. The spectrum was analyzed using the «DEIMOS» code [11] and accurate numerical values were obtained for the line energy and area, as shown in Fig. 2. The number of counts at the peak channel are also indicated in Fig. 2 to characterize the collected statistics.

The statistical errors of the line areas were typically about $10 \%$, but using the results for six intense $\gamma$ lines it was possible to obtain much better accuracy for the number of ${ }^{178 m 2} \mathrm{Hf}$ nuclei in the sample. Fortunately, no deviations were found in the relative intensity of the $\gamma$ lines as compared to the tabular values for ${ }^{178 m 2} \mathrm{Hf}$. Also, the $\gamma$-line widths were in agreement with those expected for the detector as a function of $E_{\gamma}$. These meant that no overlap occurred between the lines of interest and those from background contributions. Natural background $\gamma$ lines were definitely present in the spectrum, but did not disturb the detection of ${ }^{178 m 2} \mathrm{Hf}$. In addition, lines of ${ }^{172} \mathrm{Hf},{ }^{150} \mathrm{Eu}$ and ${ }^{133} \mathrm{Ba}$ were found in the spectrum, being long-lived enough to have survived while other radionuclides decayed completely after such a long cooling time. The area of corresponding $\gamma$ line allowed a determination of the production yield for each given isotope after taking into account the decay factors, detector efficiency and quantum yield of the line. The latter parameters were taken from the tabular data [12]. The three above-described correction factors for the detector efficiency were utilized and it 


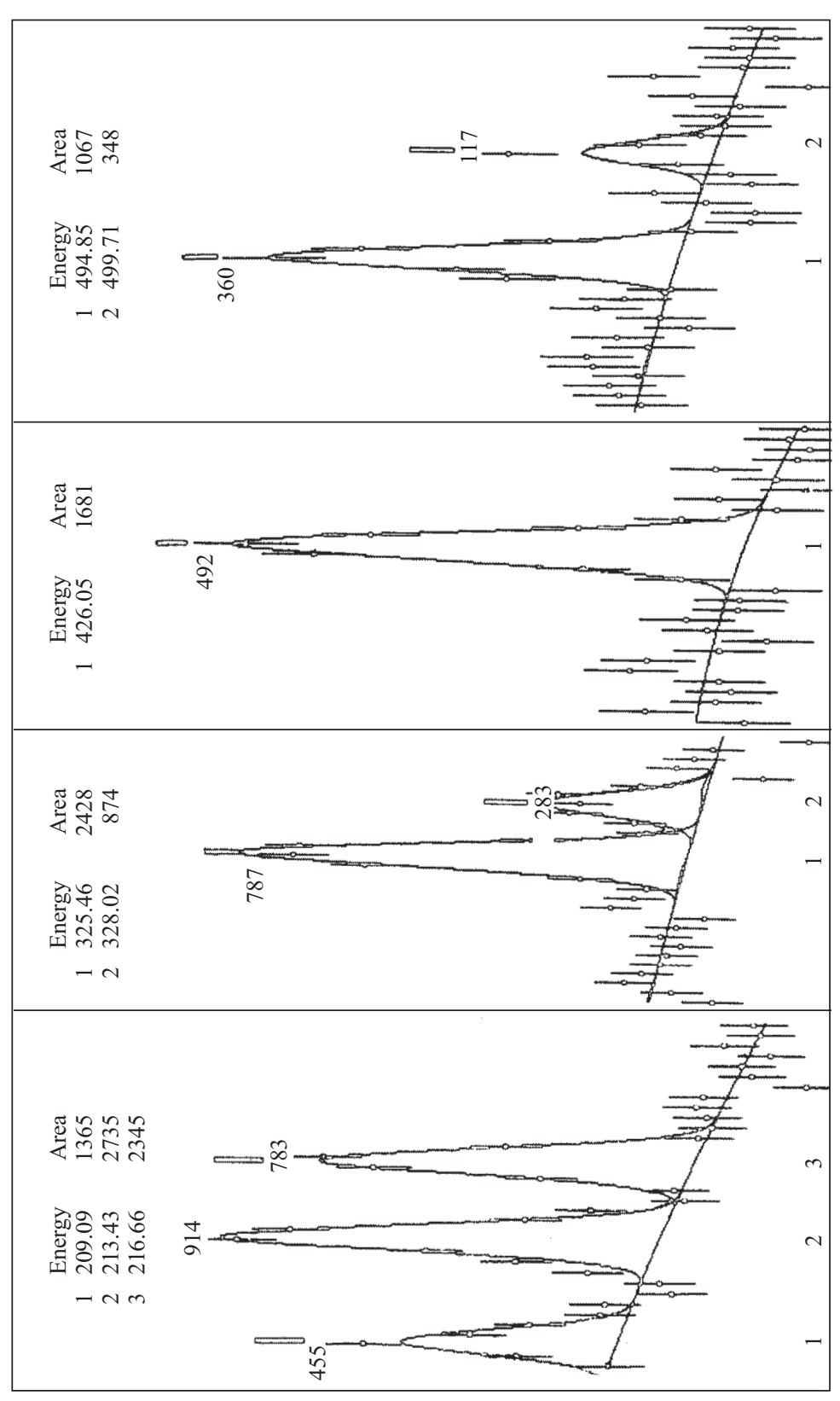

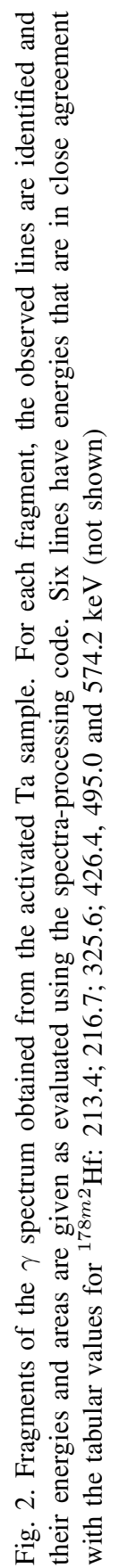


was found that the numbers of ${ }^{178 m 2} \mathrm{Hf}$ nuclei determined from different $\gamma$ lines were practically identical. The six independent measurements were averaged and the standard deviation from the mean value was found to be $5 \%$, being in agreement with the statistical errors deduced from the areas of the $\gamma$ lines. In the Table, the numbers of atoms produced by the bremsstrahlung irradiation of the Ta target are given for ${ }^{178 m 2} \mathrm{Hf},{ }^{172} \mathrm{Hf},{ }^{150} \mathrm{Eu}$ and ${ }^{133} \mathrm{Ba}$.

\section{CROSS-SECTIONS}

Having determined the number of product atoms, it was possible to evaluate the production cross-section based on a known number of projectiles reaching the target. The latter quantity was determined from the so-called «quantometer» unit, an optimized ionization chamber, that was installed in the bremsstrahlung beam for integration of the number of «equivalent» photons, $N_{\mathrm{ph}}$. This number is defined by:

$$
N_{\mathrm{ph}}=E_{\max }^{-1} \int_{0}^{E_{\max }} E_{\gamma} \frac{d N}{d E_{\gamma}}\left(E_{\gamma}\right) d E_{\gamma},
$$

for a continuous spectrum of photons with a spectral intensity $\frac{d N}{d E_{\gamma}}$ and endpoint $E_{\max }$.

The spectrum of bremsstrahlung photons emitted by electrons in matter was understood more than fifty years ago [13] and the Boethe-Heitler equation, corresponding to the ultrarelativistic case with an account of atomic screening, was used in this work. This approximation was completely satisfied in the present experiment and gives the spectral density of photons per unit electron incident on a converter:

$$
\begin{aligned}
& \frac{d \sigma}{d E_{\gamma}}=\frac{4 Z^{2} e^{4}}{137\left(m c^{2}\right)^{2} E_{\gamma}} \times \\
& \times\left\{\left[1+\left(\frac{E_{e}-E_{\gamma}}{E_{e}}\right)^{2}-\frac{2}{3} \frac{\left(E_{e}-E_{\gamma}\right)}{E_{e}}\right] \ln \left(183 Z^{-1 / 3}\right)+\frac{\left(E_{e}-E_{\gamma}\right)}{9 E_{e}}\right\} .
\end{aligned}
$$

In the present experiment, all photons generated by the converter reached the target and quantometer due to the narrow angular width of the high-energy bremsstrahlung, having a half-angle of $\theta_{0}=\frac{m c^{2}}{E_{e}} \approx 0.1 \mathrm{mrad}$. For a thick converter, the electron energy will vary considerably, so Eq. (4) must generally be integrated over $E_{e}$ as it varies from the initial incident electron energy $E_{0}$ to some $E_{\min }$. In the case of this experiment, the converter was thin and so it 
was possible to evaluate Eq. (4) without integration by using the mean value of electron energy, $\left\langle E_{e}\right\rangle<E_{0}$. Here the mean energy of electrons within the $0.3-\mathrm{mm}$ tungsten converter was determined by considering radiative losses. The spectrum of the bremsstrahlung radiation was thereby determined quantitatively for the real experimental conditions and is shown in Fig. 3. The measured number of equivalent photons supplies an absolute calibration of the spectrum. The electron beam current can also be calculated using Eqs. (3) and (4).

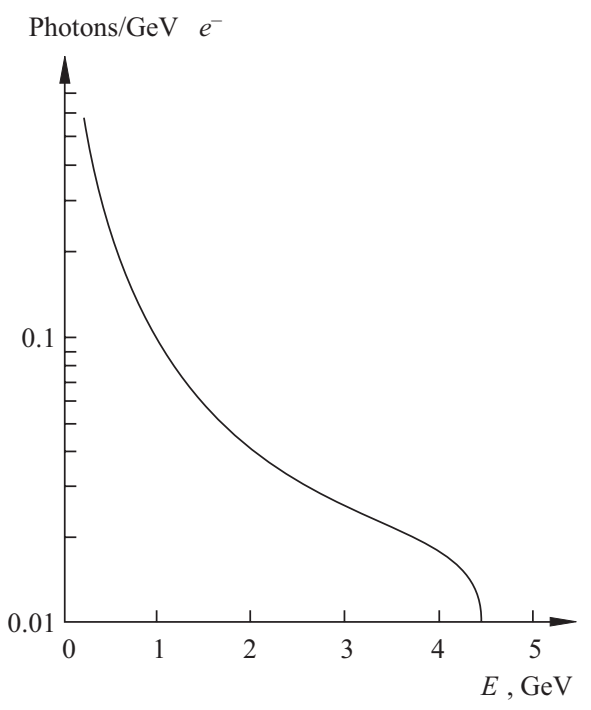

Fig. 3. Calculated and normalized spectral yield of the bremsstrahlung radiation emitted from the $0.3-\mathrm{mm} \mathrm{W}$ converter struck by the 4.5-GeV electron beam
As is mentioned above, the crosssection of photonuclear interactions demonstrates a generally decreasing trend with $E_{\gamma}$. It is quite large at $E_{\gamma}<100 \mathrm{MeV}$ due to nuclear photoabsorption and the quasi-deuteron mechanism. Then in the range of $200-800 \mathrm{MeV}$ it is enhanced again because of resonant meson generation in interactions with individual nucleons. Above 1,500 MeV the crosssection reaches an asymptotic value that is almost independent of energy, about $0.12 \mathrm{mb} /$ nucleon. In combination with the photon spectrum, shown in Fig. 3, which decreases in intensity rapidly approaching higher energies, this resulted in a drastically decreased yield, $Y_{\mathrm{nr}}\left(E_{\gamma}\right)$, of photonuclear reactions as a function of $E_{\gamma}$. The yield detected from such a reaction must occur mainly in that part of the spectrum at $E_{\gamma} \leqslant 1000 \mathrm{MeV}$, and was true for the production of the ${ }^{178 m 2} \mathrm{Hf}$ isomer. Considering the lower-energy contributions, clearly ${ }^{178 m 2} \mathrm{Hf}$ was formed in the ${ }^{181} \mathrm{Ta}(\gamma, p 2 n)$ reaction at an excitation energy above $40-50 \mathrm{MeV}$. Thus, it was assumed here that the measured ${ }^{178 m 2} \mathrm{Hf}$ yield was primarily due to photons in the range $50 \mathrm{MeV} \leqslant E_{\gamma} \leqslant 1050 \mathrm{MeV}$. The estimated inaccuracy due to this assumption, about $10 \%$, is comparable with the statistical error of these measurements.

Thus, all necessary quantities were available from which to evaluate the integral cross-section, or ICS, i. e.: the number of produced nuclei, the number of photons within the appropriate energy range and the thickness of the tantalum target. The attenuation of the bremsstrahlung flux within 2-mm target was also taken into account. The values obtained for the ICS and the mean cross-section, $\bar{\sigma}$, are given in the Table. The mean cross-section corresponds to a $1000-\mathrm{MeV}$ range 
Measured production parameters for long-lived radioactive nuclides formed after activation of a Ta target with $4.5-\mathrm{GeV}$ bremsstrahlung

\begin{tabular}{|l|l|l|c|c|}
\hline Nuclide & Type of yield & \multicolumn{1}{|c|}{$N_{\text {at }}$} & ICS $[\mathrm{MeV} \cdot$ barn $]$ & $\bar{\sigma}\left[\mathrm{cm}^{2}\right]$ \\
\hline${ }^{178 m 2} \mathrm{Hf}$ & Indep. & $(1.57 \pm 0.08) \cdot 10^{8}$ & 0.0255 & $2.55 \cdot 10^{-29}$ \\
\hline${ }^{172} \mathrm{Hf}$ & EC cum. & $(2.3 \pm 0.2) \cdot 10^{10}$ & 3.74 & $3.74 \cdot 10^{-27}$ \\
\hline${ }^{150} \mathrm{Eu}$ & Indep. & $(8 \pm 2) \cdot 10^{7}$ & 0.013 & $1.3 \cdot 10^{-29}$ \\
\hline${ }^{133} \mathrm{Ba}$ & EC cum. & $(8.7 \pm 0.9) \cdot 10^{8}$ & 0.141 & $1.41 \cdot 10^{-28}$ \\
\hline
\end{tabular}

of photon energy so that $\bar{\sigma}=\mathrm{ICS} /(1000 \mathrm{MeV})$. The products ${ }^{172} \mathrm{Hf},{ }^{150} \mathrm{Eu}$ and ${ }^{133} \mathrm{Ba}$ are also characterized in the Table. The errors in ICS and $\bar{\sigma}$ values were defined by the statistical errors in the measurements of $N_{\text {at }}$ given in the Table and systematic errors may have arisen from subsequent numerical calculations based on $N_{\text {at }}$. These systematic errors were likely to be insignificant in comparison with the statistical errors. The ${ }^{172} \mathrm{Hf}$ and ${ }^{133} \mathrm{Ba}$ nuclides accumulated the total yield of the appropriate isobaric chains, while ${ }^{178 m 2} \mathrm{Hf}$ and ${ }^{150} \mathrm{Eu}$ were only produced independently in the reactions. As expected, the mean cross-section for ${ }^{178 m 2} \mathrm{Hf}$ is lower than that known for the $p+\mathrm{Ta}$ reaction at $660 \mathrm{MeV}[5,6]$. Roughly speaking, the order-of-magnitude scale difference between these processes can be explained by the nature of the electromagnetic interaction as compared to nucleon-induced reactions.

For a physical understanding of the nuclear reaction mechanism, the isomerto-ground state ratio, $\sigma_{\mathrm{m}} / \sigma_{\mathrm{g}}$, is even more important, because it is sensitive to the angular momentum transferred to the reaction residue. Comparisons between the $\sigma_{\mathrm{m}} / \sigma_{\mathrm{g}}$ obtained from different reactions also exclude scale differences between cross sections. Unfortunately, the yield of stable ${ }^{178} \mathrm{Hf}$ ground-state nuclei could not be measured with activation techniques. The yield of ${ }^{172} \mathrm{Hf}$, however, was detected successfully and by assuming a similar ${ }^{178} \mathrm{Hf} /{ }^{172} \mathrm{Hf}$ ratio as found in the $p+$ Ta reaction [5,6] it was possible to deduce $\sigma_{\mathrm{g}}$ and therefore $\sigma_{\mathrm{m}} / \sigma_{\mathrm{g}}$ values for the bremsstrahlung-induced reaction. The latter assumption is reasonable, but there is no guaranty of its quantitatively accuracy. Therefore, only an estimate of the $\sigma_{\mathrm{m}} / \sigma_{\mathrm{g}}$ value for ${ }^{178 m 2} \mathrm{Hf}$ in the $\gamma+$ Ta reaction was possible. The inaccuracy in this value may approach $30-50 \%$. The ratio deduced in this way was

$$
\frac{\sigma_{\mathrm{m}}}{\sigma_{\mathrm{g}}} \approx 0.032,
$$

a little higher than the results of Refs. [5, 6] for ${ }^{178 m 2} \mathrm{Hf}$ formation by the $p+\mathrm{Ta}$ interaction. This is not very surprising, recalling that the $\sigma_{\mathrm{m}} / \sigma_{\mathrm{g}}$ ratio therefrom changes rather slowly with the proton energy [5,6], confirmed later in Ref. [14]. On the other hand, the similarity of isomer population in $\gamma+\mathrm{Ta}$ and $p+\mathrm{Ta}$ in- 
teractions may mean that in meson generation by photons, a significant excitation energy and momentum are transferred to the nucleus. The subsequent stages of the reaction include the emission of nucleons, the same as in the case of a nucleon interaction with a heavy target. In the end, the residual nucleus has acquired a reasonably high angular momentum. Such a conclusion is essentially reliable and is now experimentally supported.

\section{OPTIMIZATION OF PRODUCTIVITY}

In the present experiment, the converter and the target were separated and the thickness of each was relatively small. But in a view of achieving the highest possible productivity, both thicknesses should be enlarged. In this way more quanta can be created by the converter, although obviously absorption will also be increased. A reasonable compromise would be to unify both the converter and the target which would then need to be a rather thick sample of Ta. The problem of optimization of such a unified assembly was solved analytically and is described below. Figure 4 illustrates schematically the exposure of this sample configuration with an electron beam. The electron energy is reduced with thickness $t$ due to the radiation stopping power, while ionization energy loss can be neglected in the $\mathrm{GeV}$ range. The upstream part of the sample serves mostly for bremsstrahlung generation and then those photons are absorbed in the downstream part mostly due to pair production - a small fraction of the photons are absorbed due to nuclear reactions. The tracks of photons from creation to absorption are also depicted in Fig. 4. Some small fraction of them can be transmitted and leave the sample. The effective photon flux is dependent on the converter/sample thickness due to the competition between production and absorption and so the corresponding nuclear reaction yield also varies with $t$. It is necessary to calculate the optimum total thickness of the sample for the most effective production and use of bremsstrahlung, and thus to maximize the accumulation of radioactive products.

At any depth, $t$, within the sample the conversion efficiency to bremsstrahlung is proportional to the local energy of electrons, $E_{e}(t)$, because of the radiationstopping function [13]:

$$
\left(\frac{d E_{e}}{d x}\right)_{\mathrm{rad}}=c E_{e} .
$$

At the same time, the total range of energies covered by the bremsstrahlung continuum, $0 \leqslant E_{\gamma} \leqslant E_{e}$, is also compressed. For $E_{\gamma}<1050 \mathrm{MeV}$, therefore, the spectral density of radiation is almost independent of changes in $E_{e}$ in the range of 1050 to $4500 \mathrm{MeV}$ and thus different parts of the electron paths in the sample provide equal contributions to $d N_{\gamma} / d E_{\gamma}$. It was explained above that the majority of the radionuclide production was created by the part of spectrum 


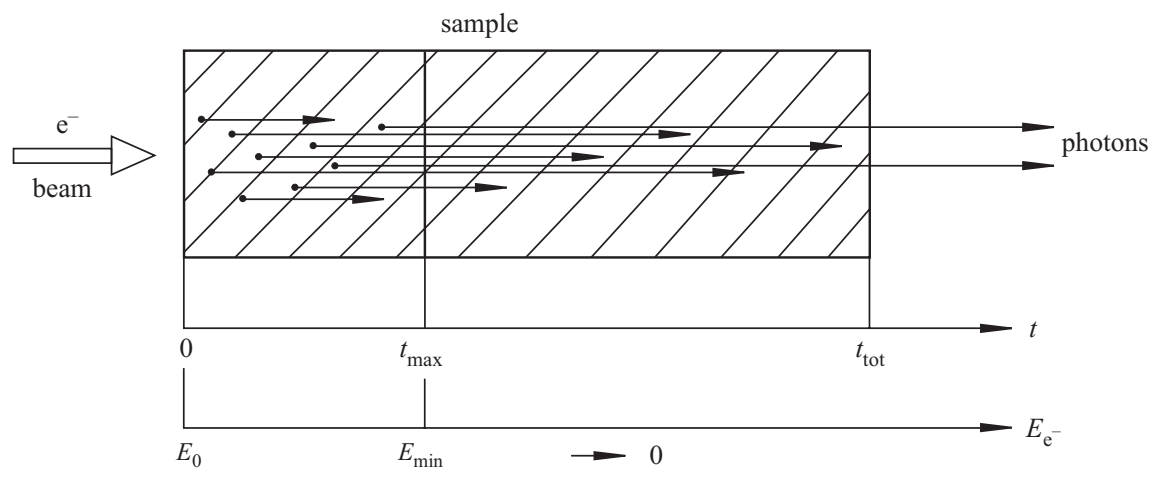

Fig. 4. Illustration of the generation and absorption of photons in a thick sample that unifies the functions of the converter and the target

from $E_{\gamma}=50-1050 \mathrm{MeV}$. The spectral density, $d N_{\gamma} / d E_{\gamma}$, and total number of photons, $N_{\gamma}$, within this range of $E_{\gamma}$ at $t=\ell$ can be expressed as an integral over the sample layer from $t=0$ to $\ell$ :

$$
N_{\gamma} \sim \int_{0}^{\ell} \frac{d E_{e}}{d x}(t) E_{e}^{-1} \mathrm{e}^{-\mu(\ell-t)} d t .
$$

With the substitution of Eq. (6) into Eq. (7), one obtains:

$$
N_{\gamma}(\ell) \sim \frac{c}{\mu}\left(1-\mathrm{e}^{-\mu \ell}\right) .
$$

Photon absorption is taken into account by a linear attenuation coefficient $\mu$ that is weakly dependent on $E_{\gamma}$ for energies greater than $100 \mathrm{MeV}$. The coefficients $c$ and $\mu$ are expressed in $\mathrm{cm}^{2}$ when the thickness values $t$ and $\ell$ are expressed in atoms $/ \mathrm{cm}^{2}$. Using Eq. (8), one deduces the total nuclear reaction yield in the sample of total thickness $d$ as:

$$
Y_{\mathrm{nr}} \sim \int_{0}^{d} N_{\gamma}(\ell) \bar{\sigma} d \ell=\frac{\bar{\sigma} c}{\mu^{2}}\left(\mu d+\mathrm{e}^{-\mu d}-1\right),
$$

where $\bar{\sigma}$ is the mean cross-section of the reaction for $E_{\gamma}$ within the range of interest.

Now, it is necessary to discuss some limiting factors on the sample thickness. After electrons penetrate through a thickness $t_{\max }$, their energy is reduced to $E_{\min }=1050 \mathrm{MeV}$ and at greater depths only softer photons can be created. The 
selected range of $E_{\gamma}$ is therefore not applicable and requiring more complicated calculations. For simplicity, here it is assumed that for depths within the sample of $t>t_{\max }$ no photons are generated, but the full thickness of the sample can produce absorption of photons and nuclear reaction events as illustrated in Fig. 4. Under such conditions the nuclear reaction yield at the total thickness of the sample is expressed as follows:

$$
Y_{\text {nr }}\left(t_{\text {tot }}\right) \sim \frac{\bar{\sigma} c}{\mu^{2}}\left[\mu t_{\max }+\mathrm{e}^{-\mu t_{\text {tot }}}-\mathrm{e}^{-\mu\left(t_{\text {tot }}-t_{\text {max }}\right)}\right] .
$$

The expression of Eq. (10) can be used to estimate the optimum thickness, $t_{\text {tot }}$, and the numerical value of the optimum yield, $Y_{\mathrm{nr}}$.

In addition to the simplifying assumptions above, the straggling of electron energy and angle was completely neglected. However, this approach may lead to an underestimation of the yield, $Y_{\mathrm{nr}}$, so Eq. (10) is still valuable for its predictive

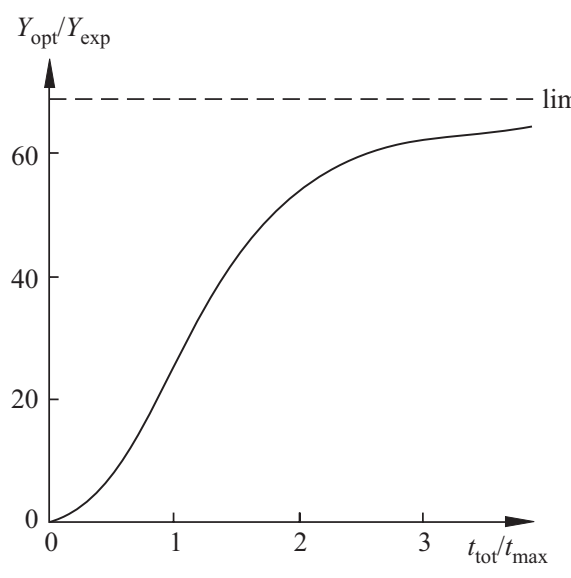

Fig. 5. Yield of nuclear reactions for production of ${ }^{178 m 2} \mathrm{Hf}$ in the unified sample, $Y_{\mathrm{opt}}$, as a function of the sample thickness. The $Y_{\text {opt }}$ value was normalized to the yield $Y_{\exp }$ taken from the experiment having separate converter and target

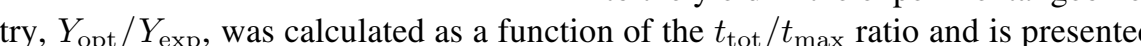
in Fig. 5. This function exhibits a growth, reaching saturation, as shown in the figure.

The value $t_{\max }$ was obtained to be of about $6.3 \mathrm{~mm}$ for a Ta sample with electron energies of $E_{0}=4.5 \mathrm{GeV}$ and $E_{\min }=1.05 \mathrm{GeV}$. As is clear from Fig. 5, there is no reason to use $t_{\text {tot }} \geqslant 3 t_{\text {max }}$. Thus, the optimum sample thickness should

power. The equations above do not contain any numerical constants by which to provide an absolute calibration of the yield. Still in this experiment and under well-defined geometry the yield was measured, and this is sufficient for the calibration. It is necessary to derive an equation corresponding to separated converter and target samples as was arranged in the experiment. Using the same theory as above, the case of separated samples is described by:

$$
Y_{\mathrm{nr}} \sim \frac{\bar{\sigma} c}{\mu^{2}}\left(1-\mathrm{e}^{-\mu \ell}\right)\left(1-\mathrm{e}^{-\mu d}\right),
$$

where $\ell$ and $d$ are the converter and the target thicknesses, respectively. Finally, the ratio of the optimized yield for the unified target-converter sample to the yield in the experimental geome-

\section{.}

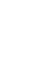

\footnotetext{
.
}

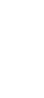

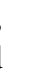


be about $19-\mathrm{mm}$ of tantalum. The absolute productivity was also estimated by linking it to the experimentally-observed production yield corresponded to the known numbers of equivalent photons and electrons incident on the converter. Finally, with a $19-\mathrm{mm}$ thick tantalum sample in unified geometry, as many as $2.5 \cdot 10^{9}$ atoms/s of the ${ }^{178 m 2} \mathrm{Hf}$ isomer may be obtained with an electron beam current of $100 \mu \mathrm{A}$. Such an intensity is achieved in modern linear electron accelerators and even higher currents may be reached in new systems that are under development.

Some of the simplifying assumptions described above do not influence the final productivity estimate due to the ability to calibrate the result with the present experimentally measured yield. Therefore, these assumptions do not serve as a source of inaccuracies in the calculated optimum value. However, two processes will be manifested within a thick unified sample which were not exhibited in the experimental conditions. First, the productivity value was evaluated in a linear approximation and so does not account for any second-order interactions, i. e. secondary radiation created by electrons and positrons after bremsstrahlung absorption with pair production. It is estimated that a correction to include secondary processes should not be higher than $15 \%$. Second, nuclear reactions induced directly by beam electrons themselves, without first their conversion to bremsstrahlung, must contribute some additional yield of the isomer within a unified converter-target assembly (recall that in the present experiment, no electrons reached the target). The yield of electronuclear reactions, $Y_{\text {enr }}$ was calculated as follows:

$$
Y_{\mathrm{enr}}=\int_{E_{1}}^{E_{2}} \frac{\bar{\sigma} k\left(E_{e}\right)}{d E_{e} / d x} d E_{e},
$$

where an integration is applied over the stopping range of electrons from energies of $E_{1}$ to $E_{2}$. The cross-section for electronuclear reactions is given by the function $\sigma_{\text {enr }}=\bar{\sigma} k\left(E_{e}\right)$, where $\bar{\sigma}$ is the photon-induced cross-section as used before. The ratio of electronuclear to photonuclear cross-sections, $k\left(E_{e}\right)$, is known for $E 1$ multipolarity to be [15]:

$$
k\left(E_{e}\right)=\frac{2 \ln \left(E_{e} / m c^{2}\right)}{137 \pi} .
$$

In reality, however, the $k\left(E_{e}\right)$ value is higher by a factor of 2.5 for $E_{\gamma}>$ $100 \mathrm{MeV}$ [15], due to such processes as $E 2$ absorption, the quasi-deuteron mechanism [16] and meson-production reactions. So, a factor of 2.5 was inserted into Eq. (13) and the result substituted into Eq. (12) prior to integration over the range of electron energies between 1050 and $50 \mathrm{MeV}$ as was done for the photon-induced reaction. The stopping range in which electron energies change from 4500 to $1050 \mathrm{MeV}$ is of less importance due to lower cross-sections and 
higher $d E_{e} / d x$, but this was nevertheless taken into account. Finally, the contribution of the electronuclear interaction was found to be about $0.13 Y_{\mathrm{phr}}$, where $Y_{\mathrm{phr}}$ was the yield determined before for the photonuclear reaction. The calculated productivity under optimum conditions was then corrected to include both secondary and electron-induced processes, giving a total yield:

$$
\sum Y_{\mathrm{opt}}=3.2 \cdot 10^{9} \text { atoms } /(\mathrm{s} \cdot 100 \mu \mathrm{A})
$$

for the ${ }^{178 m 2} \mathrm{Hf}$ isomer. This is about 8 times lower than the yield achieved at Los Alamos with a proton beam of the same current. A lower productivity by electrons compared with protons could be entirely expected. But, it is perhaps surprising that the ratio is only $1 / 8$, not by several orders-of-magnitude, and this is an important result of the experiment. One additional practical consideration should also be discussed. The volume density of power is higher in the case of electrons compared with protons due to the correspondingly larger stopping power. But this does not present a severe restriction because a multilayer tantalum sample in a water flow can effectively remove the heat released by the electron beam. It should be recalled that both radiation energy losses of electrons and photon absorption in water are negligible, as compared to Ta. In practice, it may be possible that electron beams can be more convenient and less expensive in operation than the meson-factory proton beam due to technical reasons.

\section{SUMMARY}

The yield of the ${ }^{178 m 2} \mathrm{Hf}$ isomer was measured after irradiation of a tantalum target with $4.5-\mathrm{GeV}$ bremsstrahlung. The integral and mean cross-section values and the isomer-to-ground-state ratio were newly obtained. An optimized scheme for the production of this isomer using electron accelerators was proposed and evaluated. The maximum production yield was obtained and a comparison with the productivity of proton-induced reactions was discussed.

\section{ACKNOWLEDGMENT}

The authors gratefully acknowledge support by the US Air Force Office of Scientific Research under contract F49620-02-01-0187.

\section{REFERENCES}

1. Walker P. M., Dracoulis G. // Nature. 1999. V.399. P. 35.

2. Carroll J. J. et al. // Hyperfine Int. 2001. V. 135. P. 3. 
3. Roberts H. // Hyperfine Int. 1997. V. 107. P. 91.

4. O'Brien H. A. // Nucl. Instr. Meth. B. 1989. V. 40/41. P. 1126.

5. Karamian S. A. et al. // Nucl. Inst. Meth. A. 2002. V.489. P. 448.

6. Karamian S. A., Adam J. // Czech. J. Phys. B. 2003. V. 53. P. 381.

7. Karamian S. A. et al. JINR Preprint E6-2004-7. Dubna, 2004.

8. Oganessian Y. T. et al. // J. Phys. G. 1992. V. 18. P. 393.

9. Weixiang Y. et al. // Nucl. Phys. 1992. V.14. P. 326.

10. Karamian S. A. et al. // Laser Phys. 2004. V. 14. P. 438.

11. Adam J. et al. // Measurement Techniques. 2001. V. 44. P. 93.

12. Table of Isotopes, Eighth Edition / Ed. by R. B. Firestone, V. S. Shirley, C. M. Baglin et al. New York: John Wiley and Sons, 1996.

13. Heitler W. The Quantum Theory of Radiation. Oxford: Clarendon Press, 1954.

14. Zhuikov B. L. et al. // Phys Rev. C. 2003. V.68. 054611.

15. Nedorezov V. G., Raniuk Y.N. // Part. and Nucl. 1984. V. 15. P. 379 (in Russian).

16. Levinger J. S. // Phys. Lett. B. 1979. V.82. P. 181.

Rececived on March 25, 2004. 
Корректор Т. Е. Попеко

Подписано в печать 29.04.2004.

Формат $60 \times 90 / 16$. Бумага офсетная. Печать офсетная.

Усл. печ. л. 1,18. Уч.-изд. л. 1,69. Тираж 365 экз. Заказ № 54420.

Издательский отдел Объединенного института ядерных исследований 141980, г. Дубна, Московская обл., ул. Жолио-Кюри, 6.

E-mail: publish@pds.jinr.ru www.jinr.ru/publish/ 